\title{
FORTALEZAS, DEBILIDADES Y RETOS EN LA GESTIÓN DE LA CONVIVENCIA ESCOLAR: SIGNIFICADOS PARA LOS EQUIPOS DIRECTIVOS DE ESCUELAS DE EDUCACIÓN PRIMARIA Y SECUNDARIA
}

\author{
Susana Lázaro-Visa \\ Departamento de Educación \\ Facultad de Educación. Universidad de Cantabria \\ lazaros@unican.es \\ Eva Gómez \\ Departamento de Educación \\ Facultad de Educación. Universidad de Cantabria \\ Marta García-Lastra \\ Departamento de Educación \\ Facultad de Educación. Universidad de Cantabria \\ Noelia Fernández-Rouco \\ Departamento de Educación \\ Facultad de Educación. Universidad de Cantabria
}

Fecha de Recepción: 6 Abril 2019

Fecha de Admisión: 30 Abril 2019

\section{RESUMEN}

La convivencia escolar se ha convertido en un asunto central en las políticas y prácticas educativas. Sin embargo, su sentido en los centros y los medios para promoverla se establecen y gestionan desde lógicas distintas. Este trabajo tiene por objeto comprender cómo los equipos directivos de los centros escolares significan la convivencia, qué fortalezas identifican en su gestión, así como las dificultades y retos ante los que se enfrentan. A través de un diseño exploratorio y metodología cualitativa, se realizaron 13 entrevistas semiestructuradas a representantes de equipos directivos de diferentes centros educativos de Cantabria (España). La entrevista giró en torno a los significados asociados a la convivencia y las acciones que la implican en el centro educativo, así como las posibilidades, dificultades y retos pendientes. Mediante un proceso de codificación temática se realizó un análisis comparativo de las narraciones agrupadas bajo un mismo código, incorporando los tópicos conceptual y metodológicamente consistentes. Se empleó NVivo 12 Plus. Los resultados reflejan cómo la convivencia es significada como un medio para facilitar la formación académica, pero también como un fin en sí misma, orientada al desarrollo y formación integral del alumnado, favoreciendo la construcción de una ciudadanía activa y participativa. A pesar del valor otorgado, dificultades como la poca estabilidad de las plantillas o la escasa implicación familiar destacan como 
barreras claves en el trabajo para su mejora. Como retos futuros, los equipos directivos resaltan la necesidad de formación, reflexión y análisis sobre las propuestas ya en marcha y la implicación global del centro.

Palabras clave: convivencia escolar; equipos directivos; educación primaria; educación secundaria

\begin{abstract}
Strengths, weaknesses and challenges in the management of school coexistence: significance of the management teams of primary and secondary education schools. School coexistence has become a central issue in educational policies and practices. However, its significance in schools and the means to promote it are set and administered from different approaches. The purpose of this work is to understand the ways in which school management teams can represent coexistence, the strengths they have identified in their management, as well as the difficulties and challenges they face. Through exploratory design and qualitative methodology, 13 semi-structured interviews were conducted with representatives of management teams from different schools in Cantabria (Spain). The interviews centred on the implications associated with coexistence, the actions that constitute coexistence in the educational centre, as well as the associated possibilities, difficulties and pending challenges. By means of a thematic codification process, a comparative analysis of the narratives grouped under the same code was carried out, incorporating the conceptually and methodologically consistent topics. NVivo 12 Plus was applied. The results demonstrate that coexistence is viewed as a means to facilitate academic formation, but also as an end in itself, oriented towards the development and integral formation of students, favouring the construction of an active and participatory citizenship. In spite of the value accorded, difficulties such as high staff turnover or low family involvement stand out as key barriers to improvement. In the context of future challenges, management teams emphasise the need for formation, reflection and analysis of proposals already underway and the overall involvement of the centre.
\end{abstract}

Keywords: school coexistence; smanagement teams; primary school; secondary school

La promoción de una convivencia positiva es uno de los elementos a los que prestar atención en la actualidad, como muestran los diferentes Observatorios de la Convivencia Escolar que se han puesto en funcionamiento durante los últimos años. Se refleja también en las publicaciones científicas que se han hecho eco de esta problemática, abundando cada vez más en revisiones, análisis y reflexiones que nos permitan no solo solucionar las situaciones de acoso sino tratar de prevenirlas antes de que tengan lugar, entre otros espacios, en el contexto aula (Lázaro-Visa et al., 2018). Compartimos el punto de partida de Del Rey, Ortega y Feria (2009), evidenciando que la convivencia escolar tiene que ver con el respeto en las relaciones entre todos los subsistemas que conviven en el contexto educativo. Son muchas las variables que intervienen en este entramado. Entre otras, la investigación previa pone de relieve la necesidad de promover factores personales, afectivos, morales y sociales que sabemos esenciales para el bienestar personal y las relaciones saludables con los otros (López et al., 2006; Guerra y Bradshaw, 2008). En esa misma dirección, el clima escolar en sus distintas dimensiones, parece relacionarse con menores agresiones sufridas y observadas, tanto en la infancia (Fernández-Fuertes, García-Lastra, Fernández-Rouco y Gómez, 2018) y adolescencia (Fernández-Rouco, Fernández-Fuertes, Lázaro-Visa y España, 2018), lo que subraya la necesidad de promover una cultura escolar que favorezca los procesos de vinculación, de pertenencia y satisfacción con la vida en el centro, las relaciones positivas entre los diferentes miembros 
y la percepción del centro como un lugar seguro. Con este punto de partida, nos preguntamos cómo se concretan estas prácticas en los centros, qué fortalezas identifican en su gestión y qué dificultades y retos supone para los equipos directivos su implementación en los centros.

De forma específica, el interés de este trabajo se centra en explorar la percepción de los equipos directivos en relación a lo que en sus centros se sabe, se piensa y se hace para la mejora de la convivencia, además del compromiso que el centro tiene con este asunto. Además, se profundiza en cuál es la situación de convivencial en el centro, es decir, como se articulan las relaciones entre los distintos agentes, así como las acciones que se ponen en marcha para su mejora y para la prevención de las situaciones de violencia y acoso (e.g., quién las pone en marcha, cómo).

\section{METODOLOGÍA}

En este trabajo se planteó un diseño exploratorio transversal y metodología cualitativa, que permitiera, a través del uso de entrevistas semiestructuradas, tener una perspectiva holística y profundizar en temas específicos que puedan ser comprendidos desde las propias palabras de las personas entrevistadas. Se realizaron 13 entrevistas semiestructuradas a representantes de equipos directivos de diferentes centros educativos de Cantabria (España), en concreto, tanto de ámbito rural como urbano, de carácter público y concertado, de Educación Primaria y Secundaria.

Cada entrevista giró en torno a los significados asociados a la convivencia y las acciones que la implican en el centro educativo, así como las posibilidades, dificultades y retos pendientes para la mejora de la convivencia, entre otras cuestiones no objeto de este trabajo. Las entrevistas fueron grabadas en audio y trascritas para su análisis contando con el consentimiento informado de los participantes. El procesamiento de la información se llevó a cabo utilizando el programa NVivo 12 Plus. Mediante un proceso de codificación temática, se realizó un análisis comparativo de las narraciones agrupadas bajo un mismo código, incorporando los tópicos conceptual y metodológicamente consistentes. Solo algunos de los datos más relevantes son expuestos en este trabajo.

\section{RESULTADOS Y DISCUSIÓN}

El análisis de los relatos de los equipos directivos respecto a la convivencia escolar permite explorar y repensar acerca de distintos asuntos dilemáticos en relación con dicha convivencia. En este sentido, emergen cuestiones relativas a lo que significa la convivencia para los centros educativos y las acciones que se ponen en marcha para su mejora, así como sus características y resultados, todo desde la mirada de quienes gestionan los centros educativos, es decir, alguna de las personas integrantes de los equipos directivos de los centros participantes. No hay que olvidar que el apoyo de estos equipos está relacionado con el éxito o fracaso de iniciativas para la gestión de la convivencia (Galán, Mas y Torrego, 2008).

\section{¿Cómo es significada la convivencia escolar?}

Los resultados reflejan cómo la convivencia es significada, por un lado, como un medio para facilitar la formación académica, tal y como relata una de las personas participantes: "Yo pienso que a mejor convivencia mayor aprendizaje, ¿no?", de forma consistente con trabajos previos, tanto en sentido positivo, como en sentido negativo. Es decir, la convivencia escolar positiva tiene relación con el aprendizaje y el rendimiento académico (H igaard, Kovac, Overby y Haugen, 2015), y el alumnado que es víctima de rechazo o exclusión, tiende a percibirse peor en relación a su autoeficacia académica y su rendimiento (Arslam, 2016).

Por otro lado, la convivencia también es significada por parte de los equipos directivos como un fin en sí misma, orientada al desarrollo y formación integral del alumnado, favoreciendo la construcción de una ciudadanía activa y participativa. Esta visión, tal y como se aprecia en el siguiente 
fragmento, da cuenta de cómo son las interacciones y su importancia para asegurar la igualdad de oportunidades y la participación en la vida compartida y la no vulneración de los derechos individuales (Sotomayor, Mayorga y Khal, 2018).

"Al colegio se viene a aprender y a formarnos, como personas y como... y académicamente. Tan importante 0 bastante más, seguramente, sea formarnos como personas como académicamente, van una de la mano de la otra. Y creo que esa formación académica es mucho más fácil alcanzarla a través de una buena convivencia (...)"

Sea donde sea que se coloque el foco central de la convivencia escolar, parece generalizada su consideración como esencial protagonista en el proceso educativo, entendiendo que ocuparse de ella es prioritario para el alumnado y para la gestión y satisfacción de toda la comunidad educativa, tal y como lo expresa una de las personas participantes:

"Para mí es muy importante, es muy importante porque al final yo soy... el jefe de estudios es la persona en la que terminan los problemas de convivencia que no se pueden resolver habitualmente en el aula, termina en la jefatura de estudios. Entonces, en la convivencia en el centro condiciona todo lo que se puede hacer en el centro Yo creo que si la convivencia en el centro no es adecuada, se puede... Ios alumnos pueden aprender poco, se puede esperar pocas cosas de lo que es el sistema educativo [...] tiene que haber una buena convivencia, un ambiente de convivencia adecuado, para que los profesores estén a gusto en el aula, los alumnos estén a gusto y puedan aprender, los profesores estén a gusto y puedan enseñar."

En el fragmento anterior se visibiliza también el papel que ocupan los equipos directivos. De este modo, aunque las más recientes concepciones de gestión educativa defienden menor jerarquía y más colaboración, parece que, en la práctica, sigue acudiéndose a niveles jerárquicamente superiores en el centro para la resolución de problemas convivenciales, como respuesta ante dificultades concretas y no tanto para la prevención de las mismas. Esto es central debido a la importancia que tienen los gestores educativos y sus creencias, en la dinamización de cambios en las dinámicas escolares (Murillo y Becerra, 2009).

Aun así, se subraya también en algunos momentos, la promoción del compromiso y la responsabilidad colectiva, necesarios, tal y como apunta la literatura previa (Fullam, 2009), a través de estrategias organizativas que posibiliten compartir tiempos, espacios y toma de decisiones, que permitan generar trabajo en equipo, en favor de la mejora de la convivencia, tal y como refleja una de las personas entrevistadas:

"El equipo directivo, primero, reconoce y agradece un montón la importancia, e intenta hacerle sentir importantes a todos los tutores porque es verdad que lo creemos así. Y luego por otra parte, por ejemplo, este año, como medida nueva, hemos determinado o hemos puesto en el horario de cada profesor tutor, les hemos liberado una hora lectiva para que tengan reuniones entre los tutores, que creemos que es verdad que no es suficiente, pero bueno, es una ayuda a esos tutores para que lleven a cabo su..."

\section{¿Qué forma adoptan las actuaciones para fomentar la convivencia? ¿A quién se dirigen?}

Esta preocupación por la convivencia se materializa en acciones dirigidas a todos los miembros de la comunidad educativa: profesorado, familias y fundamentalmente alumnado, si bien es necesario incidir en cómo estas son puestas en marcha, en general, de manera independiente entre sí. De este modo, no se fomenta el trabajo en conjunto, la corresponsabilidad (a pesar de que, como hemos visto, se piensa en conjunto a la hora de hablar de convivencia y de la importancia de que todas las partes participen), sino que se actúa de manera particular, por ejemplo, tal y como se recoge en una de las entrevistas realizadas, 
“...los alumnos tienen una hora a la semana que... una hora a la semana que tienen una tutoría en donde se establece, al comienzo del curso se establece un plan de... tutorial con todos los temas que se van a tratar y con un programa y todos los materiales. Entonces los padres al inicio de curso también tienen... requieren una entrevista que le informa un poco. Y luego los profesores, el equipo docente junto con el Departamento de Orientación, son los que seleccionan el material, los tutores tendrán que coordinar para la ... el seguimiento de la tutoría a lo largo de los trimestres..."

Para el alumnado, una buena parte de estas acciones es desarrollada durante las horas de tutoría, las cuales se convierten en el espacio más utilizado para abordar el fomento de la (buena) convivencia, mientras que para el profesorado se suelen organizar encuentros informales para conocerse al principio del curso, comidas, etc., como refleja uno de los participantes: "Y también tenemos, bueno, pues al final de curso, tenemos convivencias de... que nos vamos dos días, de convivencia de los profesores...". El el caso de las familias, la entrevista con el profesorado se presenta como el lugar más habitual para hablar sobre esta cuestión, "Con las familias hacemos una reunión inicial con todas las familias, pues para presentarnos, aunque la mayoría ya nos conocen, y luego llevamos un seguimiento y tenemos al menos un par de reuniones con las familias individualizadamente durante el curso".

Son diversas las formas en que se concretan las medidas que se desarrollan en los centros para fomentar la convivencia, algunas surgidas después de la aparición de problemas específicos. Entre otras, la participación en programas existentes como la Tutoría entre Iguales (TEI), Educación Responsable, el desarrollo de "tutorías individualizadas", que como señala el participante, "está funcionando así... Los niños ya empiezan "me toca a mí, ¿eh?, mañana tienes que hablar conmigo que tengo que contarte una cosa". Bueno, pues ya se han abierto un poco más"; la implementación de talleres específicos por parte de profesionales externos: "una psicóloga dando un taller, todos los martes por la tarde, con toda la clase, sobre convivencia, relaciones, asertividad, bueno, pues todo... dos trimestres estuvo". En general, encontramos un abanico de actuaciones muy amplio, desde propuestas integrales, hasta acciones puntuales, satisfactorias, pero que parecen consumir muchos recursos: "ese tipo de actividades que cuesta mucho planificarlas desde el centro, porque requiere mucha coordinación del profesorado, al final cuando termina el día te das cuenta de que bueno, pues de que ha sido otra forma de implicar al alumnado en ciertas acciones y actitudes, y que les satisface a ellos y nos satisface a nosotros".

En ocasiones, estas acciones se demandan cuando existe una situación un problema de convivencia y se debe resolver:

"Esto empezó el año pasado. La verdad que el año pasado hubo ciertos problemas de conducta, ya te digo que no eran de acoso, no era solamente dentro del centro sino también fuera del centro, y entonces nos propusieron participar con esta fundación y estamos haciéndolo. No sé si el año que viene seguirá, por nosotros sí, por supuesto, si nos ofrecen seguir, seguro que sí".

Sin embargo, los equipos directivos son conscientes de que la mejora de la convivencia es un proceso en el que es necesario seguir comprometidos y comprometidas a través del quehacer diario y la formación, como se aprecia en el siguiente fragmento:

"Bueno. Sí, hombre, yo entiendo que tienen un compromiso. ¿Sería deseable que tuvieran más? Pues seguramente sí. En general, en general los profesores del colegio asumen que, si pudiéramos desarrollar mejor las competencias socioemocionales del alumnado, pues la competencia sería mejor, y la enseñanza sería mejor. Yo creo que sí, creo que sí. ¿Y están interesados en acciones de formación? Pues yo creo que también. De hecho te decía que este año, estamos todos en un seminario de convivencia." 


\section{¿Qué dificultades y qué facilitadores emergen en la gestión de la convivencia?}

Los participantes ponen de relieve que estas actuaciones no se producen al margen del esfuerzo personal y profesional. Los grandes desafíos de la función educativa en el contexto sociocultural han puesto de manifiesto algunas dificultades, como el estrés sostenido y malestar docente que parece vincularse de forma recurrente al trabajo en los centros escolares (Llul, Martí y Brage, 2015).

En este sentido, aspectos esenciales como la carencia de tiempo suficiente, aparece como un obstáculo a la hora de poner en marcha actividades para la mejora de la convivencia: "Ojalá tuviésemos mil horas para destinar a la convivencia y para formarnos y para tratar, pero es verdad que son muchos niños, que son muchos problemas, que son muchas programaciones, que son muchos exámenes a corregir. Ojalá tuviéramos más tiempo para hacer más cosas", o para participar de una formación que facilite el proceso: "A ver, yo por ejemplo, sí que echaría de menos más actividades de formación, para nosotros, para los profesores, lo que pasa que llega un momento que tienes tantos frentes abiertos que es complicado, sobre todo en este centro, por ejemplo, porque mucha gente no es de la zona".

En otras ocasiones, los equipos directivos ponen de relieve la carencia de una visión compartida con las familias, aspecto que surge como una dificultad para promover medidas que favorezcan la convivencia, como ejemplifica uno de los equipos:

"yo, desde mi punto de vista es lo que falla, hay poco sentido de comunidad, y como comunidad educativa que somos, tenemos que participar todos, profesores, alumnos y familias. Yo no sé si es porque no sabemos, desde los centros captar esa participación de las familias"

Pero también aparece como un claro minimizador del efecto de cualquier actuación: "0 hay veces que estás intentando potenciar unos valores que a lo mejor en sus casas son diferentes, ni mejores ni peores, pero diferentes [...]".

Son múltiples las referencias en este sentido, tanto en relación a la falta de medidas conjuntas como comunidad y de continuidad entre lo que ocurre en el contexto educativo y las familias como a problemas estructurales y organizativos en los que tienen que ver distintos aspectos, como la estabilidad de la plantilla,

"lo que pasa que sí que es cierto que somos un centro con un índice de interinidad elevadísimo, el $80 \%$ del profesorado es interino, entonces trabajar con el profesorado en formación es complicado, porque tienes aquí un profesor que el año que viene puede que no esté, entonces, hasta que no haya una plantilla un poco más consolidada, es complicado trabajar en un programa que tenga continuidad. De momento sí que es, a veces tenemos la sensación de que vamos tapando parches",

También con proyectos educativos que están comenzando "lo que pasa que también el centro está, pues bueno, como hemos dicho, está arrancando". Algunas personas de la dirección ponen de relieve a su vez, la necesidad de incrementar el compromiso en el propio cuerpo docente, justificándolo, siguiendo sus relatos, en una toma de decisiones donde prima lo disciplinar "tenías que haber hecho cinco sesiones (de tutoría) y has hecho una, ¿sabes? Las sesiones son para esto y es importante. Claro, las sesiones se han hecho recortando un poco de las áreas...”, o con la carga de trabajo "tengo alguna compañera que tiene 200 alumnos, entonces al final tener esa carga de trabajo tan pesada pues te impide poder dedicar tiempo a preparar otras cosas". Como señala uno de los participantes "en el día a día es difícil por, bueno, pues por el tiempo, por el estrés, por la presión y por muchas cosas"

Cuando ponen de relieve los facilitadores, el discurso se centra fundamentalmente en las buenas relaciones existentes entre los compañeros, aunque no son atribuidas a ninguna acción específica que el centro haya podido poner en marcha para que así sea, la formación y la toma de conciencia sobre la importancia de promover una buena convivencia. También se destaca la vinculación 
entre el centro, alumnado y familias, como un eje importante: "[...] al colegio porque es como su segunda casa, digamos. Entonces, digamos, ese trato personal desde el profesorado con el alumnado y con la familia con la que van a ..., pues sí que lo valoran mucho porque es como que ellos reflejan que nos preocupamos mucho por ellos y en todos los... y en todos los ámbitos, entonces bueno"

Finalmente, hemos encontrado por parte de algunos equipos directivos una preocupación por los resultados que pone en evidencia la necesidad de diseñar procesos de evaluación de las experiencias. Estos diseños ofrecerían información a los participantes que podrían ayudarles a minimizar esa sensación de indefensión que transmiten algunas de sus afirmaciones, a la vez que mejorar las propias intervenciones. La sensación de no saber si se está logrando algo, dada la dificultad de poner en marcha procesos de evaluación de las experiencias, se refleja en las siguientes afirmaciones:

"Ahora seguimos comprometidos con ese plan de... con ese programa integral para mejorar la convivencia, vamos [...] Sí que estamos, sí que estamos trabajando en ello, aunque bueno, vamos a ver la eficacia"

"Es algo transversal, ¿no?, pero sí... sí, se recogen en el Proyecto Educativo de Centro, pero claro, al final dices, ¿lo hemos logrado, o hasta qué punto lo hemos logrado? Nos resulta difícil cuantificar hasta qué punto se logra, y si... y nos resulta difícil evaluar el logro de esta...".

\section{CONCLUSIONES}

Los resultados obtenidos ponen en evidencia diferentes aspectos. Partiendo de una visión rotunda y ampliamente compartida por parte de los equipos directivos sobre el papel clave de una convivencia positiva en el aprendizaje y desarrollo de los niños/as y adolescentes, surgen algunos elementos que nos permiten pensar en su promoción. En primer lugar, con demasiada frecuencia aparecen medidas puntuales que no parecen formar parte de una visión global, planificada, continua y de compromiso colectivo. La necesidad de continuidad en las acciones, como algo progresivo, tanto en la implicación del profesorado como en el trabajo con el alumnado, es uno de los factores que parece estar relacionado con la eficacia de las intervenciones. En segundo lugar, la propia organización de los centros, con plantillas móviles, dificulta esta continuidad. A pesar de ello, los docentes parecen ensayar distintas propuestas, sin estar plenamente convencidos de su funcionamiento, a veces con más entusiasmo, a veces con menos y casi siempre, con una carencia que parece afectar negativamente sobre su motivación: la falta de una evaluación sistemática que les permita evaluar el impacto de sus acciones o las necesidades de incorporar cambios en las mismas. Finalmente, parece que la sensación de sobrecarga en sus funciones, puede estar dificultando su compromiso para concretar en la práctica algo que, sin embargo, consideran fundamental, lo que nos permite intuir el posible malestar de un colectivo que se siente sobrepasado por las demandas, más aún cuando la actual legislación ha eliminado en las etapas de infantil y primaria la hora semanal de tutoría. La falta de tiempo y de partipación de las familias, atribuida en ocasiones, sin culpabilizar, a las propias dificultades de cada familia y en ocasiones, al no saber cómo contar con ellas, ha aparecido también como elementos sobre los que volver a pensar desde los distintos ámbitos de toma de decisiones, no solo, los equipos directivos.

\section{REFERENCIAS BIBLIOGRÁFICAS}

Arslam, G. (2016). Relationship between sense of rejection, academic achievement, academic efficacy, and educational purpose in high school students. Egitim ve Bilim, 41 (183), 293-304.

Del Rey, R., Ortega, R. y Feria, I. (2009) Convivencia escolar: fortaleza de la comunidad educativa y protección ante la conflictividad escolar. Revista Interuniversitaria de Formación del 
Profesorado, 23(3), 159-180.

Fernández-Rouco, N., Fernández-Fuertes, A. A., Lázaro-Visa, S. y España-Chico, C. (2018). Convivencia en los centros educativos: percepción del alumnado de educación secundaria obligatoria, postobligatoria y formación profesional. En S. Lázaro-Visa y A.A. Fernández-Fuertes (coord.) Clima escolar, conflicto y gestión de la convivencia en los centros educativos de Cantabria. Santander. Gobierno de Cantabria, Consejería de Educación, Cultura y Deporte, p 5796.

Fernández- Fuertes, A.A., García-Lastra, M, Fernández- Rouco, N. y Gómez Pérez, E. (2018). Convivencia en los centros educativos: percepción del alumnado de $4^{\circ}, 5^{\circ}$ y $6^{0}$ de educación primaria. En S. Lázaro-Visa y A.A. Fernández-Fuertes (coord.) Clima escolar, conflicto y gestión de la convivencia en los centros educativos de Cantabria. Santander. Gobierno de Cantabria, Consejería de Educación, Cultura y Deporte, p 29-56.

Fullan, M. (2009). Change Forces. Probing the Depths of Educational Reform. Londres: The Falmer Press.

Galán, A., Mas, C. y Torrego, J.C. (2008). Convivencia en centros educativos: investigación evaluativa en mediación y tratamiento de conflictos desde un modelo integrado. En J. C. Torrego (coord.), El plan de convivencia: Fundamentos y recursos para su elaboración y desarrollo. Madrid: Alianza Editorial.

Guerra N. G. y Bradshaw C. P. (2008) Linking the prevention of problem behaviors and positive youth development: core competencies for positive youth development and risk prevention. En N. G. Guerra y C. P. Bradshaw (eds) Core competencies to prevent problem behaviors and promote positive youth development. New directions for Child and Adolescent Development, 122, 1-17.

Høigaard, R., Kova, V. B., Øverby, N. C., y Haugen, T. (2015). Academic self-efficacy mediates the effects of school psychological climate on academic achievement. School Psychology Quarterly, 30(1), 64-74.

Lázaro-Visa, S. y Fernández-Fuertes, A. (2018) (Coord.) Clima escolar, conflicto y gestión de la convivencia en los centros educativos de Cantabria. Santander. Gobierno de Cantabria, Consejería de Educación, Cultura y Deporte.

López, F., Carpintero, E., Del Campo, A., Lázaro, S. y Soriano, S. (2006) El bienestar personal y social y la prevención del malestar y la violencia. Madrid: Pirámide.

Llull, D. S., Martí, X., y Brage, L. B. (2015). Malestar social y malestar docente: una investigación sobre el síndrome de desgaste profesional burnout y su incidencia socioeducativa. Aula, 21, 245-257.

Murillo, P., y Becerra, S. (2009). Las percepciones del clima escolar por directivos, docentes y alumnado mediante el empleo de «redes semánticas naturales». Su importancia en la gestión de los centros educativos. Revista de Educación, 350, 375-399.

Sotomayor, D. S., Mayorga, O. F., y Kahl, S. P. (2018). Convivencia escolar para la ciudadanía a la luz de las dimensiones declaradas por la UNESCO: percepción de los estudiantes de segundo ciclo. Boletín Redipe, 7(2), 94-103. 